\title{
LOCAL ISOGENY THEOREM FOR DRINFELD MODULES WITH NONINTEGRAL INVARIANTS
}

\author{
SUNGHAN BAE AND PYUNG-LYUN KANG
}

(Communicated by William W. Adams)

\begin{abstract}
An isogeny theorem for the Drinfeld modules of rank 2 over a local field analogous to that of elliptic curves is proved.
\end{abstract}

\section{0 . INTRODUCTION}

Let $k$ be a global function field over a finite constant field $\mathbf{F}_{q}$. Drinfeld introduced the notion of elliptic modules, which are now known as Drinfeld modules, on $k$ in analogy with classical elliptic curves. Hayes also studied this independently to generate certain class fields of $k$.

Drinfeld modules of rank 2 have many interesting properties analogous to those of elliptic curves. We fix $k$ to be the rational function field $\mathbf{F}_{q}(T)$. In [1] we introduced the Tate parametrization of Drinfeld modules of rank 2 with nonintegral invariants over a complete field. In this article we use the description of division points of Tate-Drinfeld modules and the methods in [6, 7] to get an isomorphism theorem for Drinfeld modules over a field with some restrictions on $t$ and $t^{\prime}$. In other words, there exist $a$ and $b$ in $A=\mathbf{F}_{q}[T]$ such that $\rho_{a}\left(t^{-1}\right)-\rho_{b}\left(t^{\prime-1}\right)$ is integral. This restriction does not appear in the classical case because $\alpha / \beta$ is a unit if the valuations of $\alpha$ and $\beta$ are equal.

From now on Drinfeld modules always mean Drinfeld modules of rank 2 defined on $A=\mathbf{F}_{q}[T]$.

\section{TATE-DRINFELD MODULES}

In this section we give a quick review of Tate-Drinfeld modules, which are the function field analogues of Tate elliptic curves [1]. Let $k=\mathbf{F}_{q}(T)$ and $k_{\infty}=\mathbf{F}_{q}((T))$, and let $C$ be the completion of the algebraic closure of $k_{\infty}$. Let $\bar{\pi}$ be an element of $C$ associated to the Carlitz module

$$
\rho_{T}=T X+X^{q} .
$$

Any rank 2 Drinfeld module $\phi$ over $C$ on $A=\mathbf{F}_{q}[T]$ is completely determined by

$$
\phi_{T}=T X+\bar{\pi}^{1-q} g X^{q}+\bar{\pi}^{1-q^{2}} \Delta X^{q^{2}} .
$$

Received by the editors April 29, 1991 and, in revised form, January 16, 1992.

1991 Mathematics Subject Classification. Primary 11G09, 11 R58.

Partially supported by KOSEF Research Grant 91-08-00-07. 
Then $g$ and $\Delta$ are modular forms on $\Omega=C-K_{\infty}$ for $\mathrm{GL}_{2}(A)$ of weight $q-1$ and $q^{2}-1$, respectively. Let

$$
t=t(z)=e^{-1}(\bar{\pi} z)
$$

where

$$
e(z)=z \prod_{a \in A}^{\prime}\left(1-\frac{z}{a \bar{\pi}}\right) .
$$

Then $g$ and $\Delta$ have $t$-expansions with coefficients in $A$ [3].

Now let $K$ be a complete field containing $k$ and $\delta>0$ a real number so that $g(t)$ and $\Delta(t)$ converge for $|t|<\delta$. For $t \in K$ with $|t|<\delta$, we define the Tate-Drinfeld module associated to $t$ by

$$
\phi_{T}^{\langle t\rangle}=T X+g(t) X^{q}+\Delta(t) X^{q^{2}} .
$$

The Tate-Drinfeld map $e_{\langle t\rangle}$ is defined to be

$$
e_{\langle t\rangle}(u)=u \prod_{a \in A}^{\prime}\left(1-\frac{u}{\rho_{a}\left(t^{-1}\right)}\right) .
$$

Remark 1.1. If one views $K$ as an $A$-module via $\rho$ (i.e., $a \cdot x=\rho_{a}(x)$ for $a \in A, x \in K)$, then $e_{\langle t\rangle}$ has exactly the same form as the exponential map $e_{\Lambda}(z)$ associated to the lattice $A \cdot t^{-1}$.

The following is given in [1].

Proposition 1.2. (i) The set $D_{t}$ of zeros of $e_{\langle t\rangle}$ is $D_{t}=\left\{\rho_{a}\left(t^{-1}\right): a \in A\right\}$.

(ii) $e_{\langle t\rangle}(u+v)=e_{\langle t\rangle}(u)+e_{\langle t\rangle}(v)$.

(iii) $\phi_{a}^{\langle t\rangle}\left(e_{\langle t\rangle}(u)\right)=e_{\langle t\rangle}\left(\rho_{a}(u)\right)$.

Remark 1.3. In the classical case, the Tate map is a homomorphism from the multiplicative group $K^{*}$ to the elliptic curve. Proposition 1.2 says that the Tate-Drinfeld map is an $A$-module homomorphism from $\bar{K}$ with $A$-module structure given by the Carlitz module to $\bar{K}$ with $A$-module structure given by the Tate-Drinfeld module $\phi^{\langle t\rangle}$.

Proposition 1.4. For $a \in A$, let $t_{a}=1 / \rho_{a}\left(t^{-1}\right)$. Then $\phi^{\langle t\rangle}$ and $\phi^{\left\langle t_{a}\right\rangle}$ are isogenous.

Proposition 1.5. Let

$$
D_{t}^{1 / a}=\left\{u \in \bar{K}: \rho_{a}(u) \in D_{t}\right\},
$$

where $\bar{K}$ is the algebraic closure of $K$. Then $e_{\langle t\rangle}$ induces a Galois isomorphism of $D_{t}^{1 / a} / D_{t}$ with $\operatorname{Ker} \phi_{a}^{\langle t\rangle}$.

\section{2. p-ADIC REPRESENTATION AND KUMMER THEORY}

Let $\mathfrak{p}=(p(T))$ be a prime ideal of $A=\mathbf{F}_{q}[T]$, where $p(T)$ is a monic irreducible polynomial in $A$. Let $\phi$ be a Drinfeld module of rank 2 . Then $\operatorname{Ker} \phi_{p(T)^{n}}$ has a natural structure of an $A / \mathfrak{p}^{n}$-module. Hence

$$
T_{\mathfrak{p}}(\phi)=\lim \operatorname{Ker} \phi_{p(T)^{n}}
$$

is an $A_{\mathfrak{p}}$-module, where

$$
A_{\mathfrak{p}}=\lim _{\longleftarrow} A / \mathfrak{p}^{n} .
$$


Let

$$
V_{\mathfrak{p}}(\phi)=T_{\mathfrak{p}}(\phi) \otimes_{A_{\mathfrak{p}}} k_{\mathfrak{p}}
$$

Now let $K$ be a finite extension of $k_{\mathfrak{p}}$ and $\phi^{\langle t\rangle}$ be a Tate-Drinfeld module of rank 2 over $K$ associated to $t$ with $|t|<1$. We use 1 instead of $\delta$ because $A$ is contained in the ring of integers of $K$ and the coefficients of $g$ and $\Delta$ are in $A$.

If $z \in D_{t}^{1 / p(T)^{n}}$, then $\rho_{p(T)^{n}}(z)$ lies in $D_{t}$. Hence there is an element $a \in$ $A$ such that $\rho_{p(T)^{n}}(z)=\rho_{a}\left(t^{-1}\right)$. The association $z \mapsto a \bmod \mathfrak{p}^{n}$ defines a homomorphism of $\Lambda_{p(T)^{n}}=\operatorname{Ker} \phi_{p(T)^{n}}^{\langle t\rangle}$ onto $A / \mathfrak{p}^{n}$. Hence the Tate-Drinfeld map gives rise to an exact sequence

$$
0 \rightarrow R_{n} \rightarrow \Lambda_{p(T)^{n}} \rightarrow A / \mathfrak{p}^{n} \rightarrow 0
$$

of $A[G]$-modules, where $G=\operatorname{Gal}(\bar{K} / K)$ and $R_{n}$ is the set of $p(T)^{n}$ th roots of $\rho$ (i.e., $\operatorname{Ker} \rho_{p(T)^{n}}$ ). By taking the limits, we obtain an exact sequence of $A_{\mathrm{p}}[G]$-modules

$$
0 \rightarrow T_{\mathfrak{p}}(R) \rightarrow T_{\mathfrak{p}}\left(\phi^{\langle t\rangle}\right) \rightarrow A_{\mathfrak{p}} \rightarrow 0
$$

and tensoring with $k_{\mathfrak{p}}$, we get an exact sequence

$$
0 \rightarrow V_{\mathfrak{p}}(R) \rightarrow V_{\mathfrak{p}}\left(\phi^{\langle t\rangle}\right) \rightarrow k_{\mathfrak{p}} \rightarrow 0
$$

where $G$ acts on $A_{\mathfrak{p}}$ and $k_{\mathfrak{p}}$ trivially.

We will show that the sequence (3) does not split. To do this we introduce an invariant $x$, which belongs to the $A$-module $\lim ^{1} H^{1}\left(G, R_{n}\right)$. Let $d$ be the coboundary map

$$
d: H^{0}\left(G, A / \mathfrak{p}^{n}\right) \rightarrow H^{1}\left(G, R_{n}\right)
$$

with respect to the sequence (1), and let $x_{n}=d(1)$. Let $x$ be an element of lim $H^{1}\left(G, R_{n}\right)$ defined by the family $\left\{x_{n}\right\}, n \geq 1$.

From the exact sequence of $A[G]$-modules

$$
0 \rightarrow R_{n} \rightarrow \bar{K} \stackrel{\rho_{p(T)^{n}}}{\rightarrow} \bar{K} \rightarrow 0
$$

we have an isomorphism $\delta: K / \rho_{p(T)^{n}(K)} \rightarrow H^{1}\left(G, R_{n}\right)$, since $H^{1}(G, \bar{K})=0$ by Hilbert's Theorem 90 .

Proposition 2.1. (a) The isomorphism $\delta: K / \rho_{p(T)^{n}}(K) \rightarrow H^{1}\left(G, R_{n}\right)$ transforms the class of $t^{-1} \bmod \rho_{p(T)^{n}}(K)$ into $x_{n}$.

(b) The element $x$ is A-torsion free.

Proof. (a) follows easily from the definition of $x_{n}$ and $\delta$. To prove (b), suppose that $a \cdot x=\rho_{a}(x)=0$ for some $a \in A$. Then

$$
a \cdot t^{-1}=\rho_{a}\left(t^{-1}\right) \in \rho_{p(T)^{n}}(K)
$$

for every $n$ by (a). Let $v$ be the discrete valuation on $K$. Then

$$
\begin{gathered}
v\left(\rho_{a}\left(t^{-1}\right)\right)=v\left(t^{-1}\right) q^{\operatorname{deg} a}, \\
v\left(\rho_{p(T)^{n}}\left(\alpha_{n}\right)\right)=v\left(\alpha_{n}\right) q^{n \operatorname{deg} p(T)} .
\end{gathered}
$$

But $\rho_{a}\left(t^{-1}\right)=\rho_{p(T)^{n}}\left(\alpha_{n}\right)$ implies that

$$
v\left(\alpha_{n}\right)=v\left(t^{-1}\right) q^{\operatorname{deg} a-n \operatorname{deg} p(T)} .
$$


But for sufficiently large $n,(4)$ implies that $v\left(\alpha_{n}\right)$ is not an integer, which is impossible.

Corollary 2.2. The exact sequence (3) does not split.

Proof. Exactly the same proof as in $[6,7]$, replacing $\mathbf{Z}_{p}$ by $A_{\mathfrak{p}}$ and $p$ by $p(T)$ would give the result.

\section{LOCAL ISOGENY THEOREM}

In this section, we will prove the following local isogeny theorem.

Theorem 3.1. Let $K$ be a finite extension of $k_{\mathfrak{p}}$ and $\mathscr{O}$ the ring of integers in $K$. Let $v$ be the discrete valuation on $K$ and $t, t^{\prime} \in K^{*}$ with $v(t)$ and $v\left(t^{\prime}\right)>0$. Let $\phi=\phi^{\langle t\rangle}$ and $\phi^{\prime}=\phi^{\left\langle t^{\prime}\right\rangle}$ be the corresponding Tate-Drinfeld modules over $K$. Suppose that there exist $a, b \in A-\{0\}$ such that $\rho_{a}\left(t^{-1}\right)-\rho_{b}\left(t^{\prime-1}\right)$ lies in $\mathscr{O}$. Then $\phi$ and $\phi^{\prime}$ are isogenous if and only if $V_{\mathfrak{p}}(\phi)$ and $V_{\mathfrak{p}}\left(\phi^{\prime}\right)$ are isomorphic as $k_{\mathfrak{p}}[G]$-modules.

Proof. The 'only if' part is trivial. To show the other direction, it suffices to show that there exist elements $\alpha, \beta \in A$ such that $\rho_{\alpha}(t)=\rho_{\beta}\left(t^{\prime}\right)$ by Proposition 1.2. Let $\varphi: V_{\mathfrak{p}}(\phi) \rightarrow V_{\mathfrak{p}}\left(\phi^{\prime}\right)$ be a $G$-isomorphism. By Corollary 2.2, $\varphi$ maps $V_{\mathfrak{p}}(R)$ into itself. After multiplying $\varphi$ by some element of $A_{\mathfrak{p}}$, we may assume that $\varphi$ maps $T_{\mathfrak{p}}(\phi)$ into $T_{\mathfrak{p}}\left(\phi^{\prime}\right)$. Then we have a commutative diagram

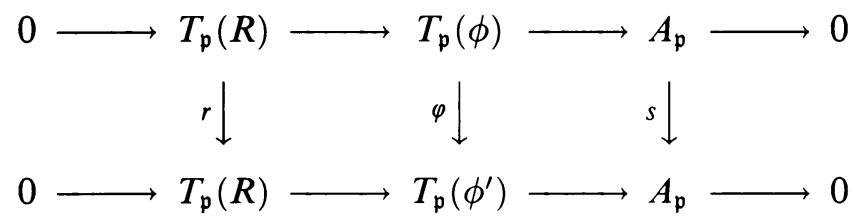

where $r, s \in A_{\mathfrak{p}}$. Let $x$ and $x^{\prime}$ be the invariants in $\lim ^{1} H^{1}\left(G, R_{n}\right)$ associated to $\phi$ and $\phi^{\prime}$, respectively, given in the previous section. Then the commutativity of (5) shows that $r \cdot x=s \cdot x^{\prime}$, that is, writing $r=\left(r_{n}\right)$ and $s=\left(s_{n}\right)$, with $\operatorname{deg} r_{n}<\operatorname{deg} p(T)^{n}$ and $\operatorname{deg} s_{n}<\operatorname{deg} p(T)^{n}$,

$$
\rho_{r_{n}}\left(x_{n}\right)=\rho_{s_{n}}\left(x_{n}^{\prime}\right)
$$

in $H^{1}\left(G, R_{n}\right)$. Therefore $\rho_{r}\left(t^{-1}\right)=\rho_{s}\left(t^{\prime-1}\right)$ in $\lim ^{\lim } K / \rho_{p(T)^{n}}(K)$ by Proposition 2.1. Let

$$
z=\rho_{a}\left(t^{-1}\right)-\rho_{b}\left(t^{\prime-1}\right) \in \mathscr{O} \text {. }
$$

Then

$$
\begin{aligned}
\rho_{s a-r b}\left(t^{-1}\right) & =\rho_{s a}\left(t^{-1}\right)-\rho_{r b}\left(t^{-1}\right)=\rho_{s}\left(\rho_{b}\left(t^{\prime-1}\right)+z\right)-\rho_{r b}\left(t^{-1}\right) \\
& =\rho_{b}\left(\rho_{s}\left(t^{\prime-1}\right)-\rho_{r}\left(t^{-1}\right)\right)+\rho_{s}(z) .
\end{aligned}
$$

Write $u=s a-r b=\left(u_{n}\right)$, with $\operatorname{deg} u_{n}<\operatorname{deg} p(T)^{n}$. Since $\rho_{s}\left(t^{\prime-1}\right)-$ $\rho_{r}\left(t^{-1}\right)=0$ in $\lim K / \rho_{p(T)^{n}}(K)$ and $\rho_{a} \rho_{b}=\rho_{b} \rho_{a}$, there exists $\alpha_{n} \in K$ such that

$$
\rho_{u_{n}}\left(t^{-1}\right)=\rho_{p(T)^{n}}\left(\alpha_{n}\right)+\rho_{s_{n}}(z), \quad v\left(\alpha_{n}\right) \leq 0 .
$$

Suppose that $u=\left(u_{n}\right) \neq 0$. Then for all sufficiently large $n$,

$$
\operatorname{gcd}\left(u_{n}, p(T)^{n}\right)=p(T)^{k}
$$


for some fixed $k<n$. Then there are $c_{n}, d_{n} \in A$ such that

$$
c_{n} u_{n}+d_{n} p(T)^{n}=p(T)^{k} \text {. }
$$

Hence

$$
\begin{aligned}
\rho_{p(T)^{k}}\left(t^{-1}\right) & =\rho_{c_{n} u_{n}+d_{n} p(T)^{n}}\left(t^{-1}\right) \\
& =\rho_{p(T)^{n}}\left(\rho_{c_{n}}\left(\alpha_{n}\right)+\rho_{d_{n}}\left(t^{-1}\right)\right)+\text { integral } \\
& =\rho_{p(T)^{n}}\left(\beta_{n}\right)+\text { integral, } \quad \beta_{n} \in K .
\end{aligned}
$$

Then $\rho_{p(T)^{k}}\left(t^{-1}-\rho_{p(T)^{n-k}}\left(\beta_{n}\right)\right)$ is integral, and so $t^{-1}-\rho_{p(T)^{n-k}}\left(\beta_{n}\right)$ is integral for all large $n$, which is impossible. Therefore $u=0$. Hence $s a=r b$ and $\rho_{s}(z)=0$ in $\lim K / \rho_{p(T)^{n}}(K)$.

Then

$$
\rho_{s_{n}}(z)=\rho_{p(T)^{n}}\left(\beta_{n}\right) .
$$

Let $k=v(s)$, the valuation of $s$ in $k_{p}$. Then $\operatorname{gcd}\left(s_{n}, p(T)^{n}\right)=p(T)^{k}$ for $n \geq k$. Hence there exist $a_{n}$ and $b_{n}$ in $A$ such that $a_{n} s_{n}+b_{n} p(T)^{n}=p(T)^{k}$.

From (6) we have

$$
\begin{aligned}
\rho_{p(T)^{k}}(z) & =\rho_{a_{n} s_{n}+b_{n} p(T)^{n}}(z)=\rho_{a_{n}}\left(\rho_{s_{n}}(z)\right)+\rho_{p(T)^{n}}\left(\rho_{b_{n}}(z)\right) \\
& =\rho_{a_{n}}\left(\rho_{p(T)^{n}}\left(\beta_{n}\right)\right)+\rho_{p(T)^{n}}\left(\rho_{b_{n}}(z)\right) \\
& =\rho_{p(T)^{n}}\left(\rho_{a_{n}}\left(\beta_{n}\right)+\rho_{b_{n}}(z)\right) .
\end{aligned}
$$

Therefore $u=\rho_{p(T)^{k}}(z)=0$ in $\lim K / \rho_{p(T)^{n}}(K)$. The proof is complete if we show that $u$ is a root of $\rho_{c}$ for some $c \in A$. Let $\mathfrak{P}$ be the maximal ideal of $\mathscr{O}$ and the residual class degree of $\mathscr{O} / \mathfrak{P}$ be $m$. Since $p(T) \in \mathfrak{P}$ and

$$
\rho_{p(T)^{n}}(X) \equiv X^{q^{n \operatorname{deg} p(T)}} \quad(\bmod (p(T)))
$$

we have

$$
\rho_{p(T)^{m}-1}(u) \equiv 0 \quad \bmod \mathfrak{P} .
$$

Let $u^{\prime}=\rho_{p(T)^{m}-1}(u)$. Then $v\left(u^{\prime}\right)>0$. Since $u^{\prime}=0$ in $\lim K / \rho_{p(T)^{n}}(K)$, there is a sequence $\left\{\delta_{n}\right\}$ in $K$ with $u^{\prime}=\rho_{p(T)^{n}}\left(\delta_{n}\right)$. Since $v \overleftarrow{v\left(u^{\prime}\right)}>0$, we have $v\left(\delta_{n}\right)>0$. In this case it is easy to see that

$$
v\left(\rho_{p(T)^{n}}\left(\delta_{n}\right)\right) \rightarrow \infty \quad \text { as } n \rightarrow \infty .
$$

Hence $u^{\prime}=\lim \rho_{p(T)^{n}}\left(\delta_{n}\right)=0$, and we are done.

Remark 3.2. The $j$-invariant $j_{t}$ of $\phi^{\langle t\rangle}$ is defined to be $j_{t}=g(t)^{q+1} / \Delta(t)$. It is shown in [3] that

$$
j_{t}=\frac{1}{t^{q-1}}+\text { power series in } t^{q-1} .
$$

Hence $j_{t}$ is nonintegral iff $v(t)>0$.

Remark 3.3. (a) The proof of Theorem 3.1 is quite similar to that of the classical case except the use of the assumption that $\rho_{a}\left(t^{-1}\right)-\rho_{b}\left(t^{\prime-1}\right)$ lies in $\mathscr{O}$. The comparison is shown in the following table: 


\begin{tabular}{|c|c|}
\hline Elliptic curve case & Drinfeld module case \\
\hline$q, q^{\prime}$ & $t^{-1}, t^{\prime-1}$ \\
\hline$v(q), v\left(q^{\prime}\right) \in \mathbf{Z}$ & $a, b \in A$ \\
\hline$\alpha=q^{v\left(q^{\prime}\right)} / q^{\prime(v(q)}$ & $z=\rho_{a}\left(t^{-1}\right)-\rho_{b}\left(t^{-1}\right)$ \\
\hline root of unity & torsion points of $\rho$ \\
\hline
\end{tabular}

In the elliptic curve case, for each element $q \in K^{*}$, there is a naturally associated integer $v(q)$, the valuation of $q$. The fact that $\alpha=q^{v\left(q^{\prime}\right)} / q^{\prime v(q)}$ is a unit in $\mathscr{O}$ is used in the proof. In our case, there is no natural element of $A$ associated to an element $t \in K$, however, we need some elements $a$ and $b$ in $A$, which make $z=\rho_{a}\left(t^{-1}\right)-\rho_{b}\left(t^{\prime-1}\right)$ to be integral in order to prove that

(i) $s a=r b$,

(ii) $z$ is a torsion point of $\rho$.

(b) The condition that $\rho_{a}\left(t^{-1}\right)-\rho_{b}\left(t^{\prime-1}\right)$ lies in $\mathscr{O}$ is not necessary if $0<$ $v(t), v\left(t^{\prime}\right)<q$. Indeed, in the proof we showed that

$$
\rho_{s_{n}}\left(t^{-1}\right)-\rho_{s_{n}}\left(t^{\prime-1}\right)=\rho_{p(T)^{n}}\left(\alpha_{n}\right)
$$

for some $\alpha_{n} \in K$ with $\operatorname{deg} r_{n}, \operatorname{deg} s_{n}<\operatorname{deg} p(T)^{n}$. Then

$$
v\left(\rho_{r_{n}}\left(t^{-1}\right)\right)=v\left(t^{-1}\right) \cdot q^{\operatorname{deg} r_{n}}>-q^{1+\operatorname{deg} r_{n}} \geq-q^{n \operatorname{deg} p(T)^{\prime}}
$$

and

$$
v\left(\rho_{s_{n}}\left(t^{\prime-1}\right)\right)=v\left(t^{\prime-1}\right) q^{\operatorname{deg} s_{n}}>-q^{1+\operatorname{deg} s_{n}} \geq-q^{n \operatorname{deg} p(T)} .
$$

Thus

$$
v\left(\alpha_{n}\right) q^{n \operatorname{deg} p(T)}=v\left(\rho_{r_{n}}\left(t^{-1}\right)-\rho_{s_{n}}\left(t^{\prime-1}\right)\right)>-q^{n \operatorname{deg} p(T)}
$$

since $v\left(\alpha_{n}\right)$ is an integer, $v\left(\alpha_{n}\right) \geq 0$. Then $\rho_{p(T)^{n}}\left(\alpha_{n}\right)$ lies in $\mathscr{O}$, as does $\rho_{r_{n}}\left(t^{-1}\right)-\rho_{s_{n}}\left(t^{\prime-1}\right)$. Hence one may take $a=r_{n}, b=s_{n}$ for any $n$.

(c) The existence of the condition prevents one from getting the global isogeny theorem. Thus one may ask: "Do there exist $a$ and $b$ so that $\rho_{a}\left(t^{-1}\right)-\rho_{b}\left(t^{\prime-1}\right)$ lies in $\mathscr{O}$ only assuming that $v(t), v\left(t^{\prime}\right)>0$ and $V_{\mathfrak{p}}(\phi)$ and $V_{\mathfrak{p}}\left(\phi^{\prime}\right)$ are $G$ isomorphic?"

Remark 3.4. One might be able to replace $A$ by a more general function ring $B$ to get the similar result. But there are some problems to be resolved primarily because $B$ is not a principal ideal domain. For example,

(i) One should consider a family of Tate-Drinfeld modules $\phi^{(\mathfrak{b})}$ for each ideal class $(\mathfrak{b})$ of $B$.

(ii) To each $\phi^{(b)}$ one must replace the Carlitz module by the sign normalized rank 1 Drinfeld module $\rho^{(\mathfrak{b})}$, which is defined over the Hilbert class field of $B$. Hence we need more restrictions on the complete field $K$ to make $\rho^{(\mathfrak{b})}$ Galois invariant.

(iii) One must define invariants of Drinfeld modules of rank 2 on $B$ to get the analogue of Proposition 1.4. 


\section{REFERENCES}

1. S. Bae and P. L. Kang, On Tate-Drinfeld modules, Canad. Math. Bull. (to appear).

2. E. Gekeler, Zur Arithmetik von Drinfeld Moduln, Math. Ann. 262 (1983), 167-182.

3. __ On the coefficients of Drinfeld modular forms, Invent. Math. 93 (1988), 667-700.

4. D. Hayes, Explicit class field theory for rational function fields, Trans. Amer. Math. Soc. 189 (1974), 77-91.

5. S. Lang, Isogenous generic elliptic curves, Amer. J. Math. 94 (1972), 861-874.

6. _ Elliptic functions, 2nd ed, Graduate Texts in Math., vol. 112, Springer, New York, Berlin, and Heidelberg, 1987.

7. J. P. Serre, Abelian $\ell$-adic representations and elliptic curves, Benjamin, New York and Amsterdam, 1968.

Department of Mathematics, Korea Advanced Institute of Science and Technology, TAEJON, 305-701, KoREA

Hongik University, Jochiwon, ChungChungnamdo, 339-800, Korea 